\title{
A case report of the orbit, ocular association and the lung in granulomatosis with polyangiitis: A diagnostic challenge
}

\author{
CHENG-WEI LU ${ }^{1}$, XIU-FEN LIU ${ }^{1}$, YING LUAN ${ }^{1}$, CHENG-BO LU $^{2}$, DAN-DAN ZHOU ${ }^{3}$, \\ LI-MIN GUO ${ }^{1}$, YA-BIN SUN ${ }^{1}$, SHENG-NAN CHEN ${ }^{1}$, YUN-LONG WU ${ }^{1}$ and JI-LONG HAO ${ }^{1}$ \\ ${ }^{1}$ Department of Ophthalmology, The First Hospital of Jilin University, Changchun, Jilin 130021; \\ ${ }^{2}$ Department of Cardiology, The First Hospital of Jiamusi University, Jiamusi, Heilongjiang 154002; \\ ${ }^{3}$ Department of Radiology, The First Hospital of Jilin University, Changchun, Jilin 130021, P.R. China
}

Received July 21,2016; Accepted April 13, 2017

DOI: $10.3892 / \mathrm{etm} .2017 .4408$

\begin{abstract}
Granulomatosis with polyangiitis (GPA) is a systemic form of vasculitis that involves small to medium sized vessels and is associated with high morbidity and mortality. GPA presents a continuous and difficult clinical diagnostic concern, due to the rarity of the disease and the diversity of the manifestations. This case report discusses the unusual symptoms presented by a particular patient, discusses these manifestations and explains how the final diagnosis was identified as GPA. A 40-year old Chinese woman was initially referred to the present institution for a progressive worsening pain, redness and gradual decrease in visual acuity in the eyes over the past 7-year period. Previous therapeutic interventions included noncompliant topical and intravenous dexamethasone for 6 years. A pre-operative examination conducted in a differing hospital to search for the presence of an orbital mass, resulted in the identification of an asymptomatic space-occupying lesion in the right middle lung, which was surgically removed in March 2015. A total of four weeks later, surgery was then applied to remove a left orbital mass, in the same hospital. A total of three months later, the patient was diagnosed with peripheral ulcerative keratitis associated with GPA, at the present institution. The corneal lesions were then treated bilaterally with cryotherapy and oral prednisone and cyclophosphamide were administered. Following surgery, the condition of the eyes appeared to be stable. A total of seven months later, the redness and pain of the right eye recurred, followed by a deep lamellar keratoplasty for the treatment of necrotizing scleritis. The condition of the two eyes was subsequently observed to be stable during the nine month follow-up. The present case study reviews various points to consider in a
\end{abstract}

Correspondence to: Dr Ji-Long Hao, Department of Ophthalmology, The First Hospital of Jilin University, Changchun, Jilin 130021, P.R. China

E-mail: 1cwny800@sina.com

Key words: granulomatosis with polyangiitis, orbital mass, lung lesions, peripheral ulcerative keratitis rare, complicated and potentially blinding case of GPA. GPA must therefore be considered in the differential diagnosis of further inflammatory conditions and tumors. Early diagnosis and an appropriate interdisciplinary approach to management, are required to decrease recurrence and morbidity in patients with GPA-mediated inflammatory ocular disease.

\section{Introduction}

Granulomatosis with polyangiitis (GPA), formerly known as Wegener's granulomatosis, is a multi-system vasculitis that preferentially involves small to medium sized vessels. The estimated annual incidence of the disease in adults is 1:100,000 (1). Its typical histologic triad consists of tissue necrosis, vasculitis, and granulomatous inflammation. If untreated, mortality, within one year from diagnosis is $90 \%$ (2). The exact etiology is still unknown. We report the unusual clinical course of a patient and the final diagnosis was identified as GPA when the pathological evaluation revealed the presence of necrotizing vasculitis and elevated serum titres of antineutrophilocyte cytoplasmic antibodies c-ANCA (anti-proteinase-3, anti-PR3). We discuss here this unusual manifestation of GPA.

\section{Case report}

A 40-year-old Chinese woman was initially referred to the clinic of Ophthalmology in July 2015 for progressive worsening pain and redness in her both eyes and gradual decrease in visual acuity during past 7 years. Past medication history included noncompliant topical tobramycin dexamethasone eye drops and intravenous dexamethasone for 6 years. The patient can't state how often and at what intervals she received dexamethasone intravenously in the past. During preoperative examination in other hospital for orbital mass, she was accidently found to have an asymptomatic space-occupying lesion in her right middle lung (Fig. 1A), which was surgically removed before removing the orbital mass in March 2015. Histopathology revealed granuloma formation with necrotizing and inflammatory cells infiltration in lung lesions (Fig. 2A). She had a surgery for removing a left orbital mass (Fig. 1B) in the same hospital 1 month later, and all blood tests were unremarkable (Table I). Histopathology revealed 
Table I. Laboratory findings.

\begin{tabular}{l}
\hline Laboratory test \\
Peripheral blood \\
Red blood cells \\
Hemoglobin \\
White blood cells \\
Neutrophil \\
Monocyte \\
Lymphocyte \\
Eosinophil \\
Basophil \\
Platelet \\
Blood chemistry \\
Total protein \\
Albumin \\
Total bilirubin \\
Glutamic-oxaloacetic \\
transaminase \\
Glutamic-pyruvic transaminase \\
Alkaline phosphatase \\
Total cholesterol \\
Blood urea nitrogen \\
Creatinine \\
Na \\
Cl \\
Ca \\
Celo
\end{tabular}

Serological tests

Erythrocyte sedimentation rate

$\mathrm{C}$-reactive protein

IgG

IgG4

IgA

$\operatorname{IgM}$

$\mathrm{C} 3$

$\mathrm{C} 4$

Rheumatoid factor

Anti-streptolysin $\mathrm{O}$ test

Proteinase 3-ANCA

Myeloperoxidase-ANCA

Anti-glomerular basement

membrane antibody

perinuclear-ANCA

cytoplasmic-ANCA

Anti-cyclic

citrullinated peptide antibody

Anti-keratin antibody

Anti perinuclear factor

HLA-B27

$4.19 \times 10^{12} / 1(3.80-5.10)$
$107 \mathrm{~g} / 1(115-150)$
$11.82 \times 10^{9} / 1(3.50-9.50)$
0.8
0.0
0.1
0.0
0.0

236x 109/1 (125-350)

$$
57.9 \mathrm{~g} / 1
$$$$
35.1 \mathrm{~g} / 1
$$

$13.7 \mathrm{umol} / \mathrm{l}(6.8-30.0)$

$16.4 \mathrm{U} / \mathrm{l}(13.0-35.0)$

$14.2 \mathrm{U} / 1(7.0-40.0)$

$67.8 \mathrm{U} / 1$ (35.0-100.0)

$5.04 \mathrm{mmol} / \mathrm{l}(2.6-6.0)$

$5.74 \mathrm{mmol} / \mathrm{l}(3.2-7.0)$

$75.6 \mathrm{umol} / \mathrm{l}$ (44-115)

$141.0 \mathrm{mmol} / \mathrm{l}$ (137-147)

$3.58 \mathrm{mmol} / \mathrm{l}(3.5-5.3)$

$102.9 \mathrm{mmol} / \mathrm{l}(99-110)$

$2.18 \mathrm{mmol} / \mathrm{l}(2.1-2.6)$

$>100 \mathrm{~mm} / \mathrm{h}(0-20)$

$66.20 \mathrm{mg} / \mathrm{l}(0-3.5)$

$9.95 \mathrm{~g} / \mathrm{l}(7-16)$

$0.176 \mathrm{~g} / \mathrm{l}(0.03-2.01)$

$2.4 \mathrm{~g} / \mathrm{l}(0.7-4)$

$1.69 \mathrm{~g} / 1(0.4-2.3)$

$1.61 \mathrm{~g} / 1(0.9-1.8)$

$0.43 \mathrm{~g} / \mathrm{l}(0.1-0.4)$

$9.19 \mathrm{IU} / \mathrm{ml}(0-15)$

$47.50 \mathrm{IU} / \mathrm{ml}(0-200)$

$<5 \mathrm{U} / \mathrm{ml}(0-5)$

$<5 \mathrm{U} / \mathrm{ml}(0-5)$

$<20 \mathrm{RU} / \mathrm{ml}(0-20)$

$$
<1: 10(<1: 10)
$$

$<1: 10(<1: 10)$

$\leq 25 \mathrm{U} / \mathrm{ml}(0-25)$

$<1: 10(<1: 10)$

(-)

$122(0-147)$

Virological test

Hepatic $\mathrm{C}$ virus antibody

Hepatitis B surface antigen
Table I. Continued.

\begin{tabular}{ll}
\hline Laboratory test & Value \\
\hline Urinalysis & \\
Protein & $(-)$ \\
Sugar & $(-)$ \\
Occult blood & $(-)$ \\
\hline
\end{tabular}

ANCA, anti-neutrophil cytoplasmic antibody; HLA, human leukocyte antigen.

hyperplasia of fibrous tissue infiltrated by dense inflammatory cells, granuloma formation and focal necrosis in the orbital mass (Fig. 2B).

On initial examination in our hospital, her visual acuity was 20/50 in both eyes. Slit lamp examination showed conjunctival congestion, and peripheral corneal infiltrates in both eyes. Fundus appearance and intraocular pressure of both eyes were normal. Blood study showed a positive immunolabeling with anti-PR3 level of 195.21 AU/ml (normal 0.00-15.00). The nephrology tests had been made and they were normal. And there were no abnormal intraoral manifestations (e.g. hyperplastic gingiva, ulcer). She was diagnosed with peripheral ulcerative keratitis associated with GPA in both eyes. The corneal lesions were then treated with cryotherapy bilaterally and with $5 \mathrm{mg}$ daily of oral prednisone and $0.1 \mathrm{~g}$ cyclophosphamide every other day. After the surgery, both eyes were stable.

Seven months later, redness and eye pain of the right eye recurred. On examination, her visual acuity was 20/200 in right eye and 20/32 in left eye. Slit lamp examination showed necrotizing scleritis and worsened peripheral ulcerative keratitis with gutter appearance from 7:00 a.m. to 10 a.m., posterior synechia of the iris at 11:00 a.m. and 2:00 p.m. in the right eye (Fig. 3A). A deep lamellar keratoplasty was preceded on her right eye in January 2016. A donor cornea graft was customized to the appropriate size and thickness and secured to the edges with buried 10-0 nylon sutures, which was epithelialized 1 week later (Fig. 3B).

Nine months later, vision was $\mathrm{CF} / 20$ inches in the right eye and 20/50 in the left eye. The conditions of both eyes were stable but blood anti-PR3 was kept at a stable, high level (148.25 AU/ml). The dosage of prednisone was increased from 5 to $10 \mathrm{mg}$ daily and that of cyclophosphamide was kept the same for $0.1 \mathrm{~g}$ every other day.

\section{Discussion}

GPA is an uncommon multi-systemic autoimmune granulomatous vasculitic disease, associated with significant morbidity and mortality. It is more prevalent in the West and rare in the East (3). Because of the diversity of the manifestations and the rarity of the disease, GPA presents a difficult clinical diagnostic problem. Thus the ophthalmologist should have a high index of suspicion and inquire about other disease-associated features, which may be symptomatic or asymptomatic. The diagnosis of our case has been difficult, we have been struggling this patient for 7 years, dealt with by pulmonologist, 

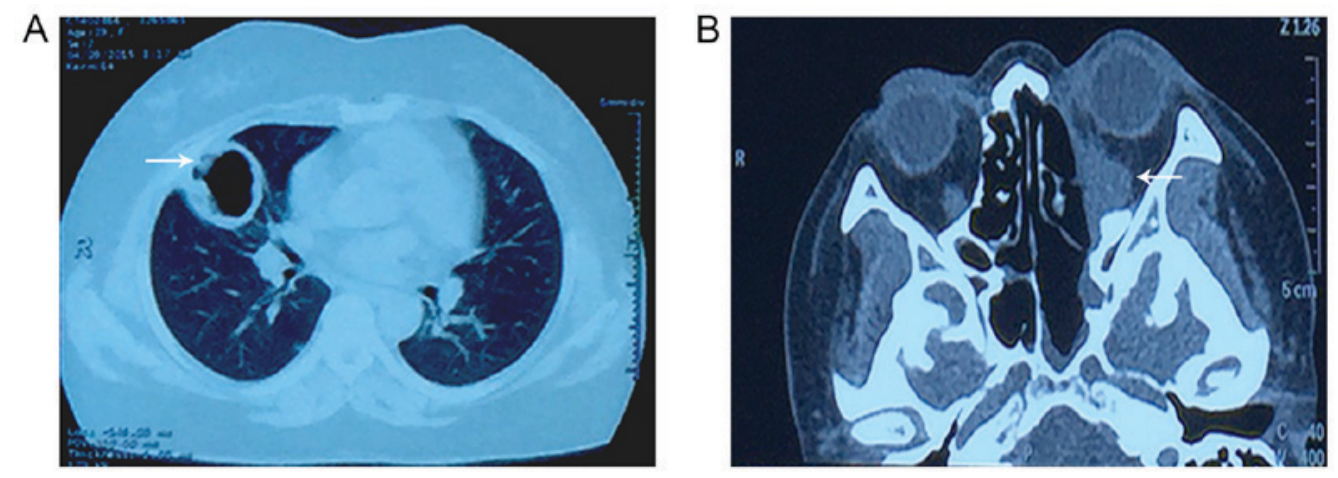

Figure 1. Montage of radiographic illustrations for left orbital mass and lung lesions. (A) The chest CT Scan showed a size of $46 \times 49 \mathrm{~mm}$ quasi-circular shaped space-occupying lesion with unevenly thickened walls and spiculated boarders in lateral segment of the middle lobe of right lung. (B) The orbital CT showed a poorly defined complex mass involving the left rectus medialis muscle and rectus inferior muscle, located in the inner lower quadrant of the left orbital outside the muscle cone.
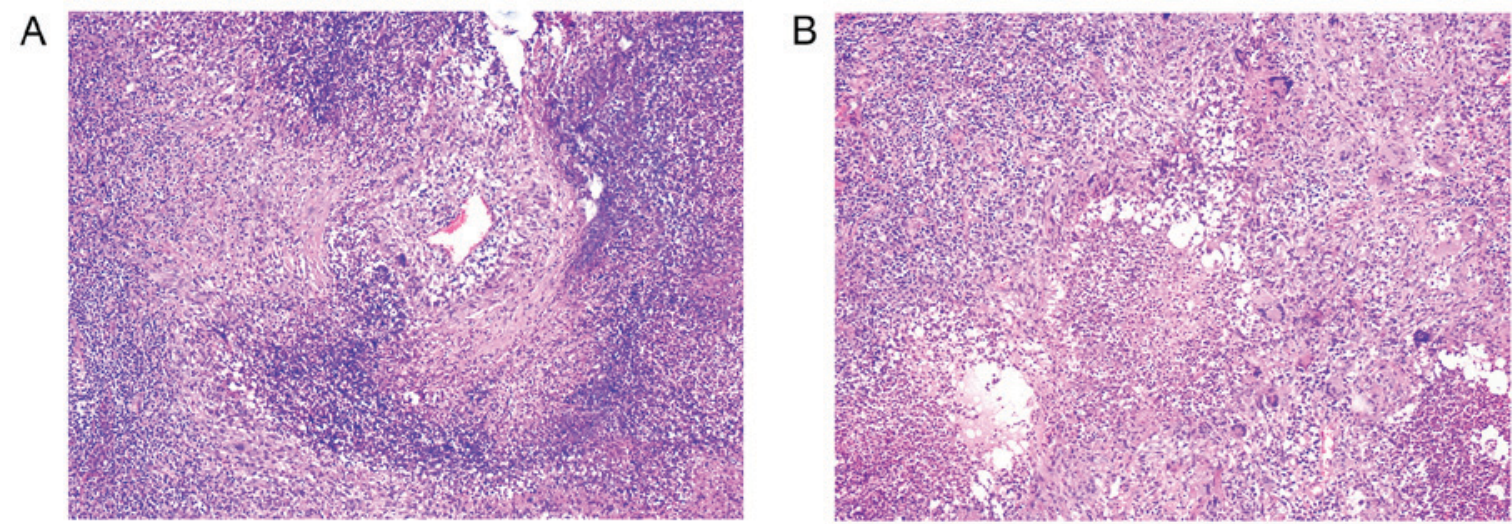

Figure 2. Montage of histopathology illustrations for left orbital mass and cavitary lesions from lung. (A) Histopathology of cavitary lesions from right middle lung showing granulomatous inflammation, necrotizing vasculitis with aggregate of inflammatory cells (H\&E staining, x200). (B) Histopathology of lesions from left orbital mass illustrated hyperplasia of fibrous tissue infiltrated by dense inflammatory cells, granuloma formation and focal necrosis (H\&E staining, $\mathrm{x} 200)$.
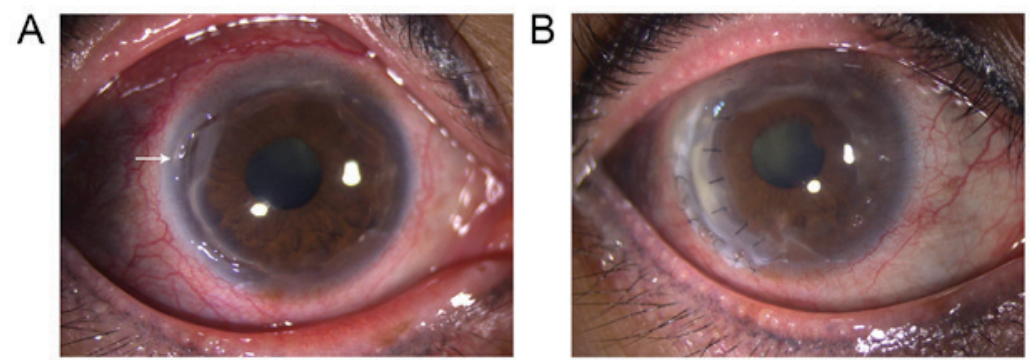

Figure 3. Montage of clinical illustrations. (A) Slit lamp examination showed peripheral ulcerative keratitis, posterior synechia of the iris and necrotizing scleritis of the right eye. (B) Slit lamp examination showed worsened healed peripheral ulcerative keratitis after deep lamellar kemtoplasty.

radiologist, ophthalmologist, and rheumatologist, and this has been an example of multidisciplinary intervention.

Orbital involvement is frequently the first or the only clinical presentation in patients with systemic or limited forms of GPA. It occurs in approximately $15-20 \%$ of GPA patients (4). Orbital GPA can cause significant morbidity and potentially lead to complete loss of vision and permanent facial deformity. Orbital disease in GPA is often difficult to distinguish from other inflammatory conditions. The differential diagnosis of orbital inflammation is broad and includes infectious, inflammatory, and neoplastic disorders. Biopsy and histopathological examination of affected tissues are important for confirming the diagnosis of WG and ruling out other diseases (5). As in our case, exophthalmos is initially present, and CT can't exclude the diagnosis of other inflammatory conditions and tumor. Histologic analysis revealed granulomatous inflammation, with areas of vasculitis in the case. Its histology is virtually indistinguishable from that of orbital inflammation and tumor.

Chest computerized tomography (CT) scans before the orbital surgery revealed pulmonary cavity in the middle lobe of right lung in our case. Clinically, these may present as cough, hemoptysis, dyspnea, or pleuritic or other forms of chest 
pain (6). But there was no known medical history of pulmonary discomfort in our case. Patients with pulmonary lesions have asymptomatic radiographic abnormalities, usually solitary or multiple pulmonary nodules with or without cavitation that may not cause symptoms in the absence of involvement of bronchi and pleura. It is hard to differentiate from lung tumor from this chest CT scan for unevenly thickened walls and spiculated boarders. Histologic analysis revealed granuloma formation with necrotizing and inflammatory cells infiltration, which is consistent with the typical histologic triad in GPA.

The American College of Rheumatology definition is the presence of two out of four of the 1990 criteria for WG, although more recent studies have included a positive ANCA immunoassay result $(7,8)$. The identification of the GPA in this case was based on a combination of abnormal orbital and chest radiograph, histologic changes and positive immunolabeling with anti-PR3.

Necrotizing scleritis and peripheral ulcerative keratitis often have a poor visual outcome in GPA. At times, however, surgical intervention is required, both for diagnosis and local control of recurrent or destructive disease. The removal of orbital mass may be successful in select cases of refractory GPA. We have reported that necrotic tissue removal, combined with cryotherapy is an effective method in the treatment of limited ophthalmic GPA (such as peripheral ulcerative keratitis and necrotizing scleritis) that was not sensitive or aggravated for traditional methods (9). However, the patient's condition recurred and became severe after 7 months. Deep lamellar keratoplasty was also reported to be effective for the treatment of PUK associated with GPA (10) and the patient's ocular condition remained stable after a deep lamellar keratoplasty.

Prompt administration of systemic immunosuppressives is the mainstay of treatment for both ocular and systemic complications in GPA. The gold standard treatment for GPA combines glucocorticoids and cyclophosphamide. More severe ocular disease is often not responsive to cyclophosphamide alone (11). Modern treatment of GPA differs between induction therapy and maintenance therapy. For induction oral or iv cyclophosphamide can be used. Normal induction therapy of oral cyclophosphamide is $1.5-2 \mathrm{mg} / \mathrm{kg}$ for at least 3 months. Cyclophosphamide should not be used for maintenance therapy. In this case a low dose of cyclophosphamide was used for a long period. The failure to induce remission might be due to suboptimal cyclophosphamide dosing, and modern guidelines do not support cyclophosphamide maintenance therapy (12). Currently there are also new therapies (e.g. Rituximab), especially concerning therapy resistance forms of GPA (2). Treatment of ocular manifestations of GPA is most successful when a multidisciplinary approach is embraced, including ophthalmologists, otolaryngologists, and vasculitis specialists (most often rheumatologists, pulmonologists, or nephrologists). Surgical intervention is indicated in the setting of locally destructive disease or for biopsy for definitive diagnosis. Concurrent systemic immunosuppressive therapy is required for long-term disease control.

In conclusion, our case highlights several important points: A rare, complicated potentially blinding case of GPA that can affect almost any organ system. It is important, however, to consider GPA in the differential diagnosis of other inflammatory conditions and tumor. Early diagnosis and an appropriate interdisciplinary approach to management are required to decrease recurrence and morbidity in patients with GPA-mediated inflammatory ocular disease.

\section{Acknowledgements}

This study was funded by the Development and Reform Commission of Jilin Province 2015Y031-1, and the First Hospital of Jilin University (grant no. JDYY72016055).

\section{References}

1. Mahr AD, Neogi T and Merkel PA: Epidemiology of Wegener's granulomatosis: Lessons from descriptive studies and analyses of genetic and environmental risk determinants. Clin Exp Rheumatol 24 (2 Suppl 41): S82-S91, 2006.

2. Bohm M, Gonzalez Fernandez MI, Ozen S, Pistorio A, Dolezalova P, Brogan P, Barbano G, Sengler C, Klein-Gitelman M, Quartier P, et al: Clinical features of childhood granulomatosis with polyangiitis (wegener's granulomatosis). Pediatr Rheumatol Online J 12: 18, 2014.

3. Chua J and Lim L: Systemic Wegener's granulomatosis with severe orbito-ocular involvement. Singapore Med J 49: e259-e262, 2008.

4. Hoffman GS, Kerr GS, Leavitt RY, Hallahan CW, Lebovics RS, Travis WD, Rottem M and Fauci AS: Wegener's granulomatosis: An analysis of 158 patients. Ann Intern Med 116: 488-498, 1992.

5. Langford CA and Sneller MC: Update on the diagnosis and treatment of Wegener's granulomatosis. Adv Intern Med 46: 177-206, 2001.

6. Tarabishy AB, Schulte M, Papaliodis GN and Hoffman GS: Wegener's granulomatosis: Clinical manifestations, differential diagnosis, and management of ocular and systemic disease. Surv Ophthalmol 55: 429-444, 2010.

7. Leavitt RY, Fauci AS, Bloch DA, Michel BA, Hunder GG, Arend WP, Calabrese LH, Fries JF, Lie JT, Lightfoot RW Jr, et al: The American College of Rheumatology 1990 criteria for the classification of Wegener's granulomatosis. Arthritis Rheum 33: 1101-1107, 1990.

8. Rao JK, Allen NB and Pincus T: Limitations of the 1990 American College of Rheumatology classification criteria in the diagnosis of vasculitis. Ann Intern Med 129: 345-352, 1998.

9. Lu CW, Zhou DD, Wang J and Hao JL: Surgical treatment of peripheral ulcerative keratitis and necrotizing scleritis in granulomatosis with polyangiitis. Saudi Med J 37: 205-207, 2016.

10. Gu J, Zhou S, Ding R, Aizezi W, Jiang A and Chen J: Necrotizing scleritis and peripheral ulcerative keratitis associated with Wegener's granulomatosis. Ophthalmol Ther 2: 99-111, 2013.

11. Masuda T, Izumi Y, Takeshita H, Kawahara C, Tsuji Y, Kurohama H, Iwanaga N, Inamoto M, Kase K, Ito M, et al: Granulomatosis with polyangiitis presenting as a choroidal tumor. Case Rep Rheumatol 2015: 271823, 2015.

12. Yates M, Watts RA, Bajema IM, Cid MC, Crestani B, Hauser T, Hellmich B, Holle JU, Laudien M, Little MA, et al: EULAR/ERA-EDTA recommendations for the management of ANCA-associated vasculitis. Ann Rheum Dis 75: 1583-1594, 2016. 